\title{
Growth, Differentiation and Secondary Metabolism in Streptomyces
}

\author{
Sulagna Roy* \\ $R$ and D Microbiology, Vadodara, Gujarat, India \\ *Corresponding Author: Sulagna Roy, R and D Microbiology, Vadodara, Gujarat, \\ India.
}

Received: February 21, 2020

Published: March 06, 2020

(C) All rights are reserved by Sulagna Roy.

\section{Introduction}

Streptomyces is a gram-positive genus of filamentous actinomycetes, a group that encompasses the majority of soil bacterial species. Actinomycetes are a large group of spore-forming bacteria that form branching filaments during growth. Streptomyces includes more than 500 species occurring in soil and several other environmental conditions. Streptomycetes evolved about 450 million years ago, have high $\mathrm{G}+\mathrm{C}$ DNA content with a complex life cycle having the potential to produce many clinically important bioactive compounds.

The Streptomyces belong to chemotype I, C (type I amino acid cell wall pattern having LL-diaminopimelic acid and glycine interpeptide bridges linking the tetrapeptides; type $\mathrm{C}$ sugar pattern lacking arabinose, galactose, xylose or madurose in whole cell hydrolysates). They usually form abundant aerial filaments bearing long chains of conidiospores, 5 to 50 or more per chain. The aerial hyphae have an extra cell-wall layer or sheath within which the hyphal tip undergoes septation to form the chain of conidia. Species and subspecies are differentiated by criteria like morphology of spore chains, spore surface, colour of aerial mycelium, colour of substrate mycelium, colour of the growth medium, physiological characteristics [1].

\section{Growth}

Growth commences when spores encounter a favourable source of nutrients and then germinate. The emerging vegetative cells, referred to as "substrate hyphae," are filamentous and not unicellular and grow by apical tip extension and branching, forming a tangled network of filamentous cells, referred to as a "substrate mycelium" [2]. It can be classified as (i) early substrate mycelium or MI and (ii) late substrate mycelium or early MII that still lacks the hydrophobic layers coating the aerial mycelium [5]. Septation may or may not occur in the substrate hyphae, with formation of either compartmentalised mycelium or of individual cells consisting of long compartments containing multiple chromosomes. Cell division is therefore dispensable for viability in streptomycetes, although it is necessary for sporulation later in their life cycle [2].

\section{Differentiation}

As the vegetative colonies age further, they produce a second filamentous cell type that grows up into the air away from the substrate hyphae (typically occurring between 24 and $48 \mathrm{~h}$ under laboratory conditions in case of Streptomyces coelicolor). These "aerial hyphae" or late MII do not branch; rather, each is fated to undergo septation events that subdivides it into 5 to 50 compartments of equal size. These "prespore" compartments, each containing a single chromosome, go on to metamorphose into spores. Spores undergo a number of maturation steps, culminating in the deposition of species or subspecies specific pigments on the spore surface that turns the aerial mycelium from white to various other colours. This aerial morphogenesis and formation of reproductive spores provide streptomycetes with a mechanism of dispersal to new environments, as they are otherwise non-motile and exhibit no apparent chemotactic behaviour [2].

The initiation of aerial hypha formation coincides with the biosynthesis of a diverse array of secondary metabolites, many of which possess significant medical application. Included among these are the majority of the medically important antibiotics, several antifungal drugs, and a variety of other important chemotherapeutic agents, such as anticancer drugs and immunosuppressants $[2]$. 


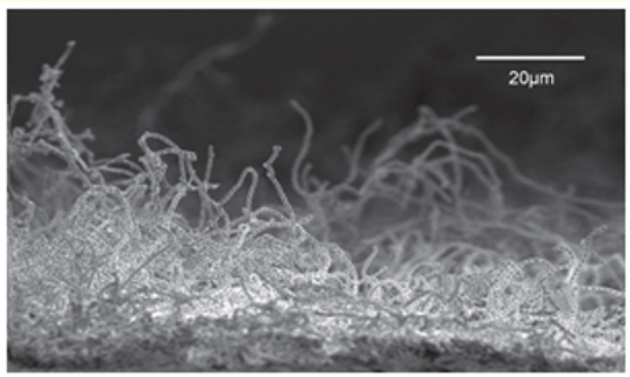

Figure 1: Scanning electron micrograph of a side-on view of a mature $S$. coelicolor colony showing long chains of spores in the air supported by a layer of substrate mycelium (photo courtesy of Kim Findlay and Mark Buttner, John Innes Centre) [2].

\section{Programmed cell death (PCD)}

Programmed cell death (PCD) is an active cellular suicide that occurs in eukaryotes and bacteria in response to both abiotic and biotic stress. In contrast to eukaryotic apoptosis, little is known about the molecular machinery that regulates bacterial PCD [6].

A process of programmed cell death has been reported early in the vegetative mycelium (as part of MI) as well as after the late substrate mycelium (after early MII) in surface cultures of Streptomyces. Several indicators, such as cell wall and membrane disruption, DNA degradation and release of the cytoplasmic content into the extracellular medium, support the existence of active, highly regulated cell suicide involving specific enzymes [7]. Calciumdependent proteolytic activation of a precursor of nucleases, the nucleolytic formation of a ladder of chromosomal bands and increased amounts of several antioxidant proteins (indicative of oxidative stress) are conspicuous events associated with the initiation of the death process $[6,7]$.

The production of $\mathrm{N}$-acetylglucosamine from peptidoglycan dismantling accelerates development and antibiotic production and might be one of the signals released during PCD [4]. Aerial mycelium would re-use nutrients released by the substrate mycelium during the second round of PCD (a kind of cannibalism) and antibiotics would be produced by the substrate and/ or aerial mycelium to prevent competition with other microorganisms during sporulation [5].

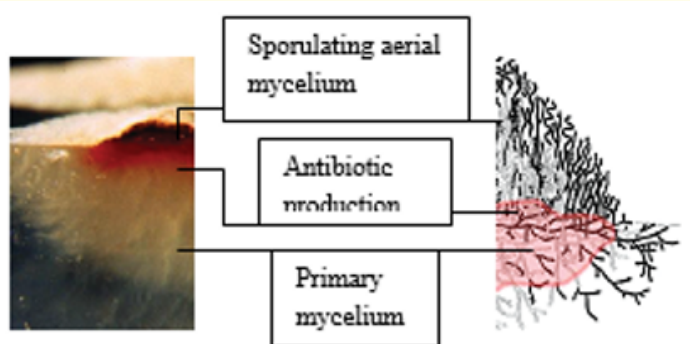

(a)

(b)

Figure 2: (a) Vertical sections through a Streptomyces colony. Photograph of colony growing on agar (courtesy of Jamie Ryding). (b) Diagram indicating how antibiotic production in the lower part of the colony can protect the nutrients released from dead cells (white) so that they can support aerial growth and sporulation. Living cells are shown as black (after Wildermuth 1970) [3].

\section{Growth, differentiation and PCD in liquid cultures}

Most streptomycetes do not sporulate in liquid cultures. Therefore, it was earlier presumed that under these conditions, there was no differentiation also. However, industrial antibiotic production is mostly performed in liquid cultures (flasks and bioreactors). Currently, it is known that in liquid cultures, differentiation is comparable to that observed in solid cultures (Figure 3). In liquid cultures, there is a first mycelium stage (MI), PCD and the differentiation of a secondary metabolite producing mycelium (MII). S. coelicolor proteomic and transcriptomic studies have shown that physiological differentiation in liquid and solid cultures is comparable. MII expresses or translates the genes involved in secondary metabolism in both solid and liquid cultures [4]. Proteins and transcripts involved in primary metabolism are up-regulated in MI, whereas proteins and genes involved in secondary metabolite biosynthesis are up-regulated in MII. The most remarkable differences between MII from solid and liquid cultures involve proteins regulating the hydrophobic cover formation and sporulation. Differentiation of MII after mycelia growth arrest is not enough to guarantee secondary metabolite production, as it can also be regulated by environmental signals, including components of the culture medium, such as nitrogen, carbon and phosphate [5]. 


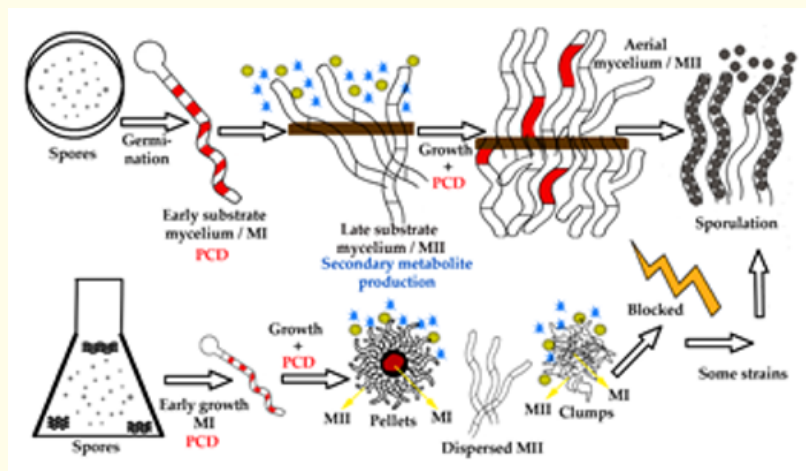

Figure 3: Streptomyces growth in solid cultures (upper panels) and liquid cultures (lower panels). In solid cultures (petri plates), spores germinate developing a compartmentalised mycelium (early substrate mycelium, MI) with $1 \mu \mathrm{m}$ average cross-membrane spacing. Some MI cells undergo a first round of programmed cell death PCD (red segments). The remaining viable segments start to grow as a multinucleated mycelium with sporadic septa (early MII, late substrate mycelium). The mycelium substrate suffers a second round of PCD(red segments) and differentiates into a mycelium that starts to grow into the air (late MII, aerial mycelium). Part of the aerial hyphae form spore chains (black circles). In liquid cultures, there is germination, MI development, PCD (in the centre of the mycelial pellets) and MII differentiation (in the periphery of the pellets). In most species, there is no aerial mycelium formation or sporulation, and hyphae form pellets and clumps. Secondary metabolites (outlined as yellow circles and blue stars) are produced by the MII hyphae [4].

However, in most Streptomyces strains, aerial mycelium formation and sporulation are blocked in liquid medium (Figure 3). On the other hand, sporulation was reported in liquid cultures for several streptomycetes, such as Streptomyces venezuelae, $S$. griseus, $S$. chrysomallus, S. antibioticus ETHZ7451, S. albidoflavus SMF301 or S. brasiliensis. Sporulation was also found to be activated in several Streptomyces species under nutritional downshifts, including the model strain S. coelicolor, and was also observed in several streptomycetes liquid cultures during the screening experiments aimed at discovering novel secondary metabolites [5].

Sporulation halts metabolism, including secondary metabolite production. Consequently, in industrial fermentations and during screening for new secondary metabolites, it is important to avoid sporulation to increase and maintain secondary metabolism for as long as possible [4].

Pellets, clumps and secondary metabolism

Pellet and clump formation have been conventionally associ- ated with secondary metabolite production in Streptomyces, but the relationship between both these processes remains obscure. Most authors have affirmed that pellets and clumps are fundamental for secondary metabolite production (e.g., retamycin in S. olindensis, nikkomycins in S. tendae, hybrid antibiotics in S. lividans), while some authors have affirmed that pellet and clump formation reduces antibiotic production (e.g., nystatin in $S$. noursei, tylosin in $S$. fradiae). One of the key events in the activation of secondary metabolite production in Streptomyces liquid cultures is the differentiation of MII. The differentiation of this mycelium is governed by PCD of the vegetative hyphae (MI), which, in liquid cultures, depends on the growth rate of the strain and hypha aggregation (pellet/clump formation) [4].

\section{Monitoring and quantification of PCD in liquid cultures}

A simple methodology based on fluorometric measures of cultures stained with SYTO9 and propidium iodide (PI) was designed to quantify PCD in liquid cultures. This method allows the efficiency of antibiotic production to be predicted based on the level of PCD. SYTO 9 is a cell-permeating nucleic acid stain which labels all of the cells, i.e., both those with intact membranes and those with damaged membranes; PI penetrates only bacteria with altered membrane permeability. Thus, in the presence of both stains, bacteria with intact membranes appear fluorescent green whereas bacteria with compromised membranes appear red, given that PI causes a reduction in SYTO 9 stain fluorescence when both dyes are present. These methodologies were applied to industrial fermentations by means of the elaboration of a protocol for the quantification of Streptomyces PCD processes. To do so, these stains were combined with fluorimetric measurements [8].

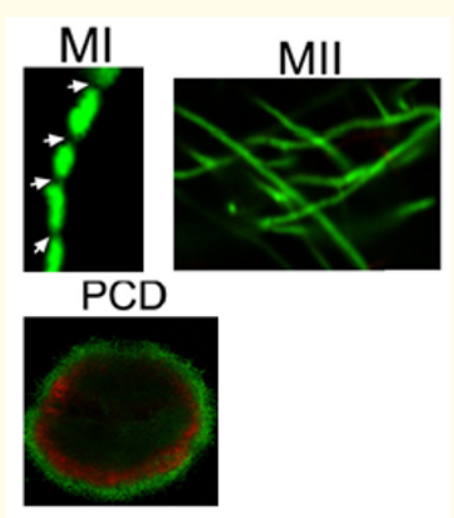

Figure 4: Confocal microscope images of key developmental stages stained with SYTO 9 and PI: individual hyphae of the first compartmentalized mycelium (MI; arrows indicate septation), second multinucleated mycelium hyphae (MII), and the mycelial pellet $(240$ $\mu \mathrm{m}$ in diameter) undergoing PCD processes in the center (red) [8]. 


\section{Conclusion}

The streptomycetes are unique as bacteria since they grow as filaments instead of unicellular forms, produce aerial spores similar to fungi and produce extremely diverse bioactive molecules. These specific features can be correlated with their complex life cycle that includes growth (involving apical tip extension, branching, karyokinesis, cytokinesis and septation), differentiation (of substrate mycelium into MI, MII, aerial mycelium formation, sporulation), programmed cell death that provides the growing aerial mycelium with soluble nutrients released by the dead cells from substrate mycelium and secondary metabolite formation that reduces competition from other microorganisms. Similar events occur even if the streptomycetes are cultured in liquid conditions. While aerial mycelium formation is lacking in submerged culture conditions, rest all other differentiation events are comparable between solid and liquid cultures.

Differentiation of MII is crucial for activation of secondary metabolism in liquid cultures of streptomycetes. MII differentiation depends on programmed cell death which in turn depends on strain specific growth rate and pellet/clump formation. Quantitative measurement of programmed cell death in liquid cultures could help in prior estimation of antibiotic/ secondary metabolite production.

\section{Bibliography}

1. Pelczar M. J. Jr., et al. "Microbiology, McGraw Hill Education (India) Private Limited". New Delhi, India, 5th edition (1993).

2. Elliot M., et al. "Chapter 24 Multicellular Development in Streptomyces, In Whitworth D (ed), Myxobacteria". ASM Press, Washington DC (2008): 419-438.

3. Chater KF. "Streptomyces inside-out: a new perspective on the bacteria that provide us with antibiotics". Philosophical Transactions of the Royal Society B 361.1469 (2006): 761-768.

4. Manteca Á and Yagüe P. “Streptomyces Differentiation in Liquid Cultures as a Trigger of Secondary Metabolism". Antibiotics (Basel) 7.2 (2018): 41.

5. Yagüe P., et al. "Pre-sporulation stages of Streptomyces differentiation: state-of-the-art and future perspectives". FEMS Microbiology letters 342.2 (2013): 79-88.

6. Manteca A., et al. "A proteomic analysis of Streptomyces coelicolor programmed cell death". Proteomics 6.22 (2006): 60086022.
7. Manteca A., et al. "Cytological and biochemical evidence for an early cell dismantling event in surface cultures of Streptomyces antibioticus". Research in Microbiology 157.2 (2006): 143-152.

8. Yague P., et al. "New method for monitoring programmed cell death and differentiation in submerged Streptomyces cultures". Applied and Environmental Microbiology 76 (2010): 3401-3404.

\section{Assets from publication with us}

- Prompt Acknowledgement after receiving the article

- Thorough Double blinded peer review

- Rapid Publication

- Issue of Publication Certificate

- High visibility of your Published work

Website: https://www.actascientific.com/

Submit Article: https://www.actascientific.com/submission.php

Email us: editor@actascientific.com

Contact us: +919182824667 